\begin{tabular}{ll} 
Revista Científica Multidisciplinaria & Ipsa Scientia \\
Vol. 4(1), 7-9 (2019) & ISSN: 2711-4406 \\
\hline
\end{tabular}

\title{
EDITORIAL
}

\section{POLÍTICA DE ARBITRAJE DE LA REVISTA Ipsa Scientia: ATRIBUTO DE CALIDAD DE LA PUBLICACIÓN CIENTÍFICA}

La expresión “arbitraje científico” (peer review, en inglés), consiste en la revisión crítica y objetiva realizada por árbitros, para evaluar los resultados de un artículo científico. Este proceso normalmente se lleva a cabo en dos fases: la primera es responsabilidad del editor o algún miembro del comité editorial, y la segunda está a cargo de dos pares o revisores externos, expertos en el campo disciplinar de la contribución postulada, a objeto de determinar si esta merece o no ser publicada en la revista.

La política editorial de la revista Ipsa Scientia declara una revisión doble ciego, que le otorga carácter completamente anónimo al proceso, toda vez que la identidad de los evaluadores y los autores se mantiene oculta durante todo el ciclo de revisiones. Los árbitros pueden sugerir la aceptación del artículo, sin cambios o condicionado a ciertas modificaciones, o en todo caso, rechazarlo. Ante posturas opuestas de los dos pares, el editor asume la decisión, o remite el artículo a un tercer árbitro para conseguir un concepto de arbitraje definitivo. Entre los criterios generales que se sugieren tomar en cuenta al momento de revisar una contribución científica, son los siguientes:

- Originalidad del contenido.

- Interés de la comunidad científica hacia el tema desarrollado.

- Aportes significativos en la disciplina de estudio.

- Grado de validez de los argumentos expresados.

- Pertinencia y calidad de las fuentes consultadas.

- Aportes del trabajo en el contexto cultural de estudio.

- Precisión y claridad de los objetivos e hipótesis.

- Coherencia de los objetivos con las conclusiones.

- Alcance y audiencia general.

- Referencias recomendadas por el autor para un mejor abordaje a las situaciones encontradas.

En este sentido, la calidad científica se articula con las buenas prácticas editoriales, de allí que la política de Ipsa Scientia también declara la necesidad de un comportamiento ético, de acuerdo con los principios de transparencia establecidos a nivel internacional, tomando en cuenta los siguientes aspectos:

IPSA SCIENTIA - Vol. 4 Nº 1 - 2019 
El editor y el equipo editorial deben actuar de forma justa, objetiva y sin ningún tipo de discriminación; se comprometen a publicar los trabajos basados principalmente en sus méritos académicos, o sea, sin tener en cuenta ningún tipo de influencia comercial o conflicto de intereses, y cumplir con la política de privacidad. La decisión tomada por el editor, cualquiera que fuese esta, será basada en el concepto de los pares revisores; ante la sospecha de plagio, el comité editorial se valdrá de herramientas de software disponible.

En cuanto a los revisores, estos deben contribuir al proceso de evaluación de los artículos emitiendo conceptos objetivos, libres de juicio de valor, respetuosos, constructivos y oportunos. Asimismo, deben manifestar si tienen conflicto de interés relacionado con el trabajo o los financiadores del mismo que los incline a la aceptación o rechazo del artículo. Actuarán bajo los criterios de competencia, confidencialidad, imparcialidad y honestidad, diligencia, respeto y cortesía. Se comprometen en llevar a cabo una revisión crítica, honesta, constructiva y objetiva tanto de la calidad científica como de la calidad literaria de la contribución.

Bajo este esquema de directrices, tengo el orgullo de presentar el contenido del volumen 4 de la revista, el cual nos presenta en primer lugar, el artículo original de Paternina, Linares y Hernández titulado Transferencia de tecnología y conocimiento en el sector exportador de bovinos, que busca formular una estrategia de transferencia de tecnología y conocimiento aplicada al sector mencionado en la ciudad de Barranquilla, Colombia. Los resultados evidencian muy alta presencia de la dimensión tipos de estrategias; alta presencia de las modalidades de transferencia y las etapas de adquisición; y mediana presencia de los mecanismos de transferencia; por ello los autores concluyen que la propuesta constituye un aporte para el desarrollo de una cultura tecnológica y el fomento de la inteligencia competitiva en las empresas exportadoras de ganado bovino de Barranquilla.

En segundo lugar, el trabajo realizado por los autores Ruiz, García y Martínez, titulado Gestión ambiental para la innovación y competitividad de las organizaciones ecoturísticas en áreas protegida, cuyo propósito fue analizar la gestión ambiental como estrategia para la innovación y competitividad en estas organizaciones prestadoras de servicios ecoturísticos en el Parque Nacional Natural Tayrona. Los resultados ofrecen una percepción de estas organizaciones sobre la gestión ambiental y el desarrollo de su actividad productiva; las estrategias y herramientas ambientales utilizadas en el sector ecoturístico. Se concluye acerca de la aplicación continua de estrategias ambientales preventivas e integradas a los procesos productivos, los productos y los servicios con el fin de incrementar la eficiencia, competitividad y rentabilidad de las organizaciones.

Seguidamente, Quintana, Ramos y Lugo nos ofrecen su trabajo Riesgo biológico generado por el manejo de residuos sólidos en un centro hospitalario; con el objetivo de evaluar el riesgo biológico generado por el manejo de residuos sólidos por parte del personal de servicios generales en la E.S.E Hospital Local Cartagena de Indias. Los resultados obtenidos demuestran que las actividades donde los trabajadores presentan mayor contacto con agentes biológicos son en el lavado de camillas, cambio de bolsas rojas para residuos peligrosos y lavado de guardián, siendo esta última inusual. Se concluye que las medidas preventivas y de seguridad que adopta el personal en su puesto de trabajo son eficientes con relación al mantenimiento de la limpieza y en

IPSA SCIENTIA - Vol. 4 No $1-2019$ 
orden, y son conscientes de la exposición a agentes biológicos. Por ello, se debe realizar capacitación en autocuidado y uso adecuado de los elementos de protección personal, como acciones preventivas para evitar accidentes o enfermedades laborales.

Por su parte, Ahumada, Palacio, Posada y Orjuela estudiaron la Percepción del riesgo laboral en trabajadores operativos del sector metalmecánico. Para ello, diseñaron un protocolo para la percepción del riesgo laboral con énfasis preventivo en accidentes y enfermedades laborales para las empresas del sector metalmecánico de la ciudad de Barranquilla. Los principales hallazgos indican que los trabajadores presentan un bajo nivel en prevención de accidentes y enfermedades laborales; se evidencia la falta de intervención del gobierno y pocos recursos de los empleadores para cumplir con la normatividad.

Otra contribución de interés es la investigación de Fong, Taron y Zabaleta quienes exponen su cisión sobre el Nuevo liderazgo organizacional para fortalecer instituciones universitarias débilmente acopladas según Weick, donde analizaron la institución universitaria del siglo XXI desde la perspectiva de Weick (1976) y cómo el débil acoplamiento entre sus estamentos incide en sus procesos organizacionales desde la alta dirección hacia las unidades académicas y departamentos. Se concluye que la flexibilidad en el funcionamiento de las universidades modernas se garantiza por la débil articulación de sus elementos constituyentes que potencializan su efectividad, por el alto índice de iniciativa con el que cuentan en el modelo universitario y a la capacidad de solicitar colaboración del resto de las unidades universitarias, lo que permite identificar la no linealidad del sistema jerárquico universitario. Asímismo unificar el liderazgo transaccional y transformacional universitario en un solo liderazgo como el liderazgo directivo con características del nuevo liderazgo de Burns, permitirá transformar la organización universitaria en Meta-Universidad con altos estándares de calidad

Finalmente, presentamos el trabajo de Rojas y Murcia, titulada Salud visual y ocular de escolares atendidos en una clínica universitaria oftalmológica de Cartagena de Indias (Colombia), con el objetivo de caracterizar la salud visual y ocular de los niños en las entidades atendidas por la Fundación CharityVisión y Universidad del Sinú en la ciudad de Cartagena. Se analizaron estadísticamente los resultados y se plantearon recomendaciones que permitirán dar una mejor calidad de vida a todas las personas que lo requieren. Se concluye que es de vital importancia implementar las políticas públicas en estos casos como el programa 'Quiero Ver' los cuales deben existir para poder invertir más en la población vulnerable.

Es interés superlativo del equipo editorial de la revista Ipsa Scientia el publicar artículos científicos con calidad; de esta manera, construimos un prestigio ganado dado el cumplimiento de una política transparente para seguir consolidándonos como vitrina del conocimiento y la "ciencia misma". 